\title{
Posibilidades y limitaciones del aprendizaje cooperativo en el EEES: análisis de una experiencia en el aula de inglés ${ }^{1}$
}

\author{
Paula López Rúa \\ Universidad de Santiago de Compostela
}

Recibido: 30 octubre 2013 / Aceptado: 20 abril 2014

ISSN: $1697-7467$

\begin{abstract}
RESUMEN: Este estudio pretende comprobar el funcionamiento del aprendizaje cooperativo en la educación superior a través del análisis de una actividad inspirada en la técnica del juego-concurso por equipos ideada por D. DeVries $(1973 ; 1974)$ y llevada a cabo en una asignatura de inglés dentro del grado de Maestro de Educación Primaria. Se concluye la conveniencia de impulsar este tipo de aprendizaje en la formación universitaria, aunque la disminución de la presencialidad, el elevado número de alumnos y su deficiente formación previa en cuanto a responsabilidad en el aprendizaje, planificación y hábitos para operar en grupos pueden limitar la implantación exitosa del EEES.

Palabras clave: aprendizaje cooperativo, EEES, juego-concurso, inglés
\end{abstract}

Possibilities and Limitations of Cooperative Leaning in the EHEA: Reporting on an Experience in the FL Classroom

\begin{abstract}
The aim of this study is to check the functioning of cooperative learning in higher education by reporting on an activity carried out in an English course in the new degree in Primary School Teaching. The activity is partially based on the TGT (TeamsGames-Tournaments) technique devised by D. DeVries (1973; 1974). The conclusions point at the convenience of promoting this kind of learning in university education. However, the study also reveals that the successful implementation of the EHEA may be hindered by the decrease in the hours of classroom instruction, large class sizes, and the students' poor training as regards their responsibility for learning, planning habits and efficient team-work. Keywords: cooperative learning, EHEA, TGT, English
\end{abstract}

\section{INTRODUCCIÓN: EL EEES Y EL APRENDIZAJE COOPERATIVO}

La implantación del EEES ha traído consigo una serie de retos metodológicos para el profesorado, tales como focalizar la docencia en la adquisición de competencias y destrezas, fomentar el trabajo autónomo, y prestar atención personalizada a través de las tutorías académicas. El seguimiento continuado y la atención personalizada se acompañan, no obstante, de una disminución de la presencialidad en las aulas, a la que hay que sumar, en el caso de determinados grados o asignaturas, el aún elevado número de alumnos por aula.

\footnotetext{
${ }^{1}$ Este trabajo ha sido subvencionado por el Ministerio de Economía y Competitividad (FFI2012-31450) y la Consellería de Educación de la Xunta de Galicia (CN2012/81).
} 
Estos factores dificultan de modo evidente la labor del docente, que en muchos casos trata de cumplir con los requisitos del nuevo sistema evaluando al alumnado mediante la asignación de trabajos de grupo. En apariencia, estos trabajos facilitan la evaluación de un grupo numeroso de alumnos, se adaptan adecuadamente a la reducción de la presencialidad, ya que el alumnado puede trabajar fuera del aula y ser supervisado en tutorías, y fomentan el trabajo autónomo y la responsabilidad individual, al darse por sentado que el alumnado es capaz de realizar un reparto adecuado de tareas y responsabilidades dentro del grupo.

A este respecto, en el informe de la segunda fase del proyecto Tuning (González y Wagenaar, 2006) se revisan ocho competencias genéricas, incluyendo medios y actividades para desarrollarlas y evaluar su adquisición en diferentes áreas disciplinares. El "trabajo de grupo" aparece explícitamente mencionado como "medio ligado al desarrollo" de las denominadas "habilidades interpersonales" (2006: 242), y concretamente en el área de Educación, la capacidad para trabajar en grupo se considera de hecho una habilidad interpersonal: "en Educación, las habilidades interpersonales se definen de manera que incluyan (...) la capacidad de trabajar en grupo" (2006: 243). No obstante, no se hace ninguna referencia al modo en que se desarrolla o debería desarrollarse ese trabajo en grupo, y es evidente que no todos los formatos de trabajo de grupo contribuyen de la misma manera a la adquisición de habilidades interpersonales ni reflejan adecuadamente la tarea y el proceso de aprendizaje llevados a cabo por cada individuo.

El informe del proyecto Tuning reconoce que las habilidades interpersonales, consideradas esenciales en áreas como Educación o Enfermería, han sido tradicionalmente descuidadas en la educación superior, a pesar de su importancia para los futuros graduados independientemente de su área de especialización. Simplemente se ha dado por supuesto que "los alumnos irían 'haciéndose' con las apropiadas habilidades interpersonales conforme se hicieran adultos" (2006: 244). Por otra parte, las áreas que las consideran esenciales también señalan que los estudiantes "deberían poseer y poseen con toda seguridad" muchas de esas habilidades al iniciar sus estudios, aunque al mismo tiempo reconocen la necesidad de aportar "elementos esenciales" o incluso "volver enteramente a moldearlas" (2006: 243). Esta afirmación parece indicar que son habilidades que el alumnado debería haber desarrollado en otras etapas educativas, aunque en la práctica no lo hace o lo hace de forma inadecuada.

Es precisamente en el ámbito de las habilidades interpersonales en donde las técnicas de aprendizaje cooperativo se manifiestan como una herramienta que puede contribuir de modo eficaz a su adquisición, puesto que se orientan específicamente al correcto desarrollo de las capacidades para el trabajo grupal. La denominación "aprendizaje cooperativo" abarca un conjunto heterogéneo de técnicas de trabajo en grupo que se llevan aplicando en las aulas en diferentes materias y etapas educativas ya desde mediados de los años 60 del pasado siglo, aunque en nuestro país comenzaron a alcanzar una cierta popularidad a partir de los años 90. El resultado de este interés ha sido la publicación de un creciente número de contribuciones sobre el tema, tanto de orientación más teórica (Ovejero, 1990; García, Traver y Candela, 2001; Goicoetxea y Pascual, 2002) como de carácter didáctico, esto es, informes de experiencias llevadas a cabo en variadas materias de diferentes etapas educativas: por ejemplo, Cano (2012) y Bernabé (2012) en educación infantil y música en educación primaria, respectivamente; Geronès y Surroca (1997) en lengua y la literatura, Lorenzo y otros (2002) en TICs, o Serrano y otros (2008) en ciencias sociales, todos ellos en educación secundaria; o, ya en estudios universitarios, Fraile (2008) en educación físi- 
ca, Area y Martín (2009) en matemáticas para ingeniería de telecomunicaciones, Andreu y Sanz (2010) en "Fundamentos de cognición humana" (asignatura perteneciente al grado en Documentación e Información), López Rúa (2010) en lenguas extranjeras; o Barba, Martínez y Torrego (2012) en "Dimensión pedagógica y procesos educativos" (asignatura del grado de Maestro/a de Educación Infantil).

A pesar de la variedad y las características particulares de las distintas técnicas cooperativas, todas ellas comparten una filosofía basada en cinco principios (Johnson, Johnson y Holubec, 1994):

1. La interdependencia positiva de los miembros del grupo. El docente presenta una tarea en la que todos los miembros se necesitan para alcanzar un objetivo común. El éxito o fracaso es conjunto, no individual, lo cual fomenta el interés de todos los miembros por que sus compañeros aprendan lo máximo posible. Si cada uno lleva a cabo la tarea que le corresponde, su trabajo contribuirá al éxito conjunto.

2. La responsabilidad grupal e individual. Como el resultado depende del trabajo de todos, el grupo asume unos objetivos comunes. Al mismo tiempo, a cada individuo le corresponde una tarea específica de la que es responsable, de modo que el trabajo individual contribuye decisivamente al éxito del grupo.

3. La interacción estimuladora. El alumnado trabaja en el aula cara a cara, y la función del profesor es esencialmente de asesoramiento y supervisión. Disminuye por tanto la interacción alumno-profesor en favor de la interacción alumno-alumno.

4. El desarrollo de actitudes, valores y habilidades individuales y grupales. Los miembros del grupo aprenden a trabajar juntos, a administrar recursos y manejar conflictos, pero también a ayudarse y apoyarse, y a superar prejuicios, complejos, hostilidades y egocentrismos.

5. La evaluación grupal. Para premiar el trabajo conjunto y la cooperación, y conseguir el compromiso de todos los miembros con el grupo, se procura que la evaluación de resultados sea en gran medida conjunta en lugar de individual, de modo que todos los miembros comparten la misma calificación.

Existen evidentes coincidencias entre los fundamentos del aprendizaje cooperativo y algunos principios en los que se basa el EEES. Así, por ejemplo, en el EEES se propone que el proceso educativo pase a centrarse en el alumno, que se hace responsable de su aprendizaje, y que necesita desarrollar actitudes y habilidades personales e interpersonales no sólo para su vida académica, sino también para su futura vida profesional. Las técnicas de aprendizaje cooperativo fomentan ese desarrollo, y colocan al alumnado en el centro del proceso y en un papel activo, mientras que el profesor, en un segundo plano, ejerce como planificador, asesor y supervisor. Con el aprendizaje cooperativo, el alumnado aprende además a trabajar por sí mismo y a solucionar problemas, sabiendo que ya no puede depender exclusivamente de un profesor que antes seleccionaba, explicaba e interpretaba íntegramente el material. Este aspecto puede relacionarse con el interés desde el EEES por proporcionar herramientas que estimulen el aprendizaje autónomo y continuado. En definitiva, y tal y como señalan, entre otros, León del Barco y Latas (2007), el aprendizaje cooperativo puede responder eficazmente a las demandas de este nuevo escenario educativo, lo cual apunta a la conveniencia de impulsar este tipo de aprendizaje en la formación universitaria: 
El nuevo Espacio Europeo de Educación Superior supone un desplazamiento de una educación centrada en la enseñanza hacia una educación centrada en el aprendizaje, a la vez que se exigen a los alumnos nuevas competencias interpersonales que faciliten la interacción social y la cooperación. No nos cabe la menor duda de que el aprendizaje cooperativo, dentro de este nuevo contexto, va ser [sic] una metodología que todo profesor universitario debe conocer y aplicar en las aulas universitarias (León del Barco y Latas, 2007: 272-73).

En esta misma línea se manifestaba también Escribano (1995), en cuyo análisis del aprendizaje cooperativo y autónomo en la educación superior sostiene que la formación de individuos cooperativos es una meta, a la vez que un desafío, para la enseñanza universitaria:

(...) uno de los retos actuales sería llegar a ser un estudiante o profesor/a cooperativo. Sólo se cambia en comunidad, interpelándose mutuamente y trabajando en equipo en línea de aprendizaje cooperativo. Unos estudiantes-profesores/as cooperativos son un desafio. Y la formación universitaria que se implique en lograr este desarrollo comunitario tendrá que revisar seriamente sus prácticas y sus actuaciones (Escribano, 1995: 93).

Teniendo en cuenta lo anteriormente expuesto, el presente trabajo tiene como objetivo comprobar el funcionamiento de determinadas prácticas de aprendizaje cooperativo en el entorno universitario, verificando su idoneidad dentro de los planes de estudio adaptados a Bolonia y poniendo de manifiesto posibles problemas en la adaptación a los nuevos grados. Para ello se puso en marcha la actividad que se describe en el siguiente apartado. Dicha actividad fue precedida por una experiencia piloto realizada en una asignatura de perfil equivalente perteneciente a la antigua diplomatura de maestro.

\section{Análisis de la aCtividad CoOperativa}

\subsection{Objetivos}

Como ya se ha mencionado, el objetivo de la actividad es poner en práctica los principios del aprendizaje cooperativo, esto es, fomentar la responsabilidad individual y grupal, la interacción cara a cara y el desarrollo de habilidades personales e interpersonales, y premiar la cooperación del grupo recurriendo a la evaluación conjunta. Mediante la técnica utilizada (ver apartado siguiente) se pretende conjugar la interacción cooperativa con la competitiva, introduciendo en el aprendizaje un aspecto lúdico que podría incrementar la motivación y el rendimiento. Se considera además como objetivo el aumento de la autoestima del alumnado, ya que el comprobar que cada uno tiene algo que aportar consigue que se sientan útiles y se valoren más a sí mismos. Por otra parte, se consideró que la realización de esta actividad para adquirir contenidos gramaticales relacionados con la lengua extranjera podía favorecer la retención de los mismos, puesto que los sujetos básicamente pasivos en una sesión expositiva se convierten en activos al tener que cooperar en grupos, revisar y practicar lo aprendido, y enseñar a otros. Se espera por tanto un aumento general del rendimiento en términos de capacidad de retención y aplicación de contenidos. 


\subsection{Técnica empleada: adaptación del juego-concurso por equipos}

Una de las técnicas de aprendizaje cooperativo más conocidas y exitosas es el juegoconcurso por equipos (en inglés TGT o Teams-Games-Tournaments) ideado por D. DeVries y otros colaboradores como K. Edwards y R. Slavin (véanse, por ejemplo, DeVries y Edwards, 1973; DeVries y Edwards, 1974; o Slavin, 1986). En términos simples, se trata de un método que combina el aprendizaje puramente cooperativo con el componente competitivo. El profesor divide al alumnado en grupos heterogéneos y presenta el material de estudio. Posteriormente, los alumnos trabajan en equipos cuyos miembros se preparan entre ellos asegurándose de que todos dominan el tema lo mejor posible. Hasta este punto, la técnica coincide con el método "Aprender juntos" (Learning Together) desarrollado por los hermanos Roger y David Johnson (véase, por ejemplo, Johnson y Johnson, 1994). No obstante, el juego-concurso añade el elemento competitivo a través de un torneo. Los alumnos del mismo nivel de todos los equipos se enfrentan entre ellos respondiendo a preguntas de ese nivel y obteniendo puntos para su equipo. Finalmente, se suman los puntos acumulados y todos los miembros del equipo ganador reciben la misma recompensa.

Como se detalla más adelante, la actividad piloto realizada en la diplomatura se llevó a cabo siguiendo el procedimiento anteriormente descrito, en una clase de 25 estudiantes. No obstante, el elevado número de alumnos y la menor duración de la asignatura en el grado supusieron un importante problema para el docente a la hora de preparar material para la competición y formar equipos de heterogeneidad fiable, por lo que la competición por niveles fue reemplazada por preguntas de niveles elegidos al azar que responderían todos los miembros de cada equipo.

\subsection{Contexto de aprendizaje}

La actividad se llevó a cabo durante el curso académico 2011-2012 en la asignatura "Enseñanza y Aprendizaje de Competencias Comunicativas: Inglés" (EAI), asignatura obligatoria del grado de Maestro de Educación Primaria, impartida en la Facultad de Formación del Profesorado del Campus de Lugo (Universidad de Santiago de Compostela). Las características generales de la asignatura se detallan en la Tabla 1:

Tabla 1. Asignatura para la puesta en práctica de actividad cooperativa

\begin{tabular}{|c|c|}
\hline Asignatura & EAI \\
\hline Curso y duración & $2^{\text {o } \text { curso, semestral }}$ \\
\hline $\begin{array}{c}6 \text { créditos ECTS } \\
\text { Créditos y horas }\end{array}$ & $\begin{array}{r}51 \text { horas presenciales: } 24 \text { expositivas, 24 interactivas (3 grupos) y } \\
3 \text { de tutorías (6 grupos) }\end{array}$ \\
\hline Alumnos & 63 \\
\hline $\begin{array}{c}\text { Niveles de competencia } \\
\text { (entrada y salida) }\end{array}$ & $M C E R^{2}$ B1 a B2 (B.1.1. a B.2.1.) \\
\hline
\end{tabular}

${ }^{2}$ Marco Común Europeo de Referencia para las Lenguas 
Por lo que respecta al nivel de competencia comunicativa, la asignatura persigue la consolidación del nivel B1 con las especificaciones descritas en el MCER (2002: 30-31).

\subsection{Desarrollo de la actividad: preparación, presentación y realización}

La actividad se llevó a cabo en varias fases: preparación del profesor, presentación de la actividad a los grupos, y realización (preparación de los equipos y concurso)

\subsubsection{Formación de los equipos}

Para formar los equipos de trabajo se utilizaron las divisiones ya establecidas en tres grupos interactivos de 20, 25 y 18 alumnos, constituyéndose en total 14 equipos de 4 ó 5 alumnos. La figura del "ayudante" (assistant) se introdujo, junto con la penalización por falta de asistencia (ver sección 2.4.7.), para solucionar dos inconvenientes que podían afectar a la composición de los equipos y a la marcha de la experiencia: la imposibilidad de formar equipos con el mismo número de alumnos y el absentismo ocasional de sus miembros. El ayudante, elegido dentro de cada equipo y con obligatoria asistencia a todas las sesiones, tiene la función de cubrir bajas ocasionales para que todos los equipos cuenten con el mismo número de participantes en la competición. A fin de asegurar su participación en el trabajo del grupo de no haber bajas, se establecieron también normas de reemplazo obligatorio.

Los equipos fueron organizados por el profesor procurando una combinación heterogénea de miembros en diferentes aspectos: competencia lingüística, género, y hábitos de agrupación en el aula. No obstante, y debido al escaso tiempo disponible, la configuración del programa y el elevado número de alumnos, su fiabilidad en la práctica se vio comprometida al tener que basarse únicamente en la observación durante un mes del comportamiento en el aula, la calificación más reciente obtenida en lengua inglesa aportada por el alumnado, y una calificación de referencia obtenida en un test de gramática de tres niveles que el alumnado realizó individualmente fuera del aula.

\subsubsection{Diseño de las preguntas}

El docente diseñó una batería de 330 preguntas organizadas en 3 grados de dificultad para tres rondas de competición sobre los siguientes temas del programa de contenidos gramaticales: Passive constructions (construcciones pasivas), Modal verbs (verbos modales) y Conditions and wishes (condiciones y deseos). Cada alumno responde a dos preguntas de dificultad dispar al azar (grados 1 y 2, 1 y 3, ó 2 y 3). Los ejemplos (1) a (3) corresponden a preguntas de los tres grados sobre el último tema:

1. Grado 1: Oil (float) if you (pour) it on water $\rightarrow$ Oil floats if you pour it on water

2. Grado 2: I didn't tell him the truth. I regret it. I wish ... $\rightarrow$ I wish I had told him the truth

3. Grado 3: I know his story is true because I was there. I (not believe) it if I (not see) it with my own eyes $\rightarrow$ I wouldn't have believed it if I hadn't seen it... 


\subsubsection{Temporalización}

La actividad se desarrolló en 8 horas de clase y 6 sesiones, a razón de 2 sesiones por tema. En la primera sesión, el profesor realizaba una presentación 30-40 minutos, a la que seguía 1 hora de preparación de los equipos en el aula con el material suministrado por el profesor (teoría, ejercicios y soluciones). La segunda sesión comprendía unos 15 minutos de repaso y 40-45 minutos de concurso. Al margen de la preparación dentro del aula, los equipos podían continuar su preparación fuera de la misma el tiempo que considerasen conveniente.

\subsubsection{Trabajo en equipo y concurso}

En la actividad desarrollada en la diplomatura, tras la sesión de preparación cada equipo decidía qué miembro concursaría en cada nivel para competir por niveles. En el caso del grado, y debido a las limitaciones ya apuntadas, una vez realizadas las sesiones de preparación en grupo, el profesor formula preguntas de diferentes niveles mezcladas al azar. Los miembros de cada equipo deciden su turno de participación y todos ellos responden a las preguntas.

El sistema empleado en la diplomatura seguía más de cerca la técnica original (aunque en esta última la asignación de niveles se hacía obligatoriamente a partir de los resultados de la ronda anterior). La asignación voluntaria presenta ciertas ventajas: cada miembro se hace responsable de su aprendizaje eligiendo en qué nivel desea competir, y tiene la posibilidad de medirse a otros que elijan su mismo nivel de dificultad, lo cual fija una meta alcanzable que puede incrementar su motivación y autoestima. Por otra parte, se fomenta la autonomía, ya que el equipo organiza a sus participantes y toma decisiones sobre quién compite. Entre los inconvenientes, no obstante, se encuentra la tendencia al encasillamiento. El sistema empleado en el grado, por su parte, presenta el inconveniente del factor azar pero evita los encasillamientos y en teoría fuerza al equipo a trabajar más unido, ya que todos los participantes pueden tener que responder a preguntas de cualquier nivel, lo cual estimula la preparación conjunta en lugar de la "especialización" en un nivel.

\subsubsection{Premios}

Se destinaron a esta actividad 0,5 puntos sobre 5 por actividades de aula y, para recompensar el trabajo general de todo el alumnado, se estableció un sistema decreciente de puntuación para todos los equipos, de 0,5 a 0,1 puntos, no permitiendo empates.

\subsubsection{Tiempo de dedicación del profesor}

Un aspecto a veces pasado por alto en los informes de experiencias didácticas es la estimación de carga de trabajo adicional que dichas experiencias implican para el docente, y que en ocasiones supone otro inconveniente para la realización de este tipo de actividades de innovación educativa. En este caso, las horas de dedicación del profesor fuera del aula para la puesta en marcha de la actividad fueron aproximadamente 34. En la Tabla 2 se desglosan las diferentes tareas del profesor y una estimación de horas de dedicación a cada tarea: 
Tabla 2. Dedicación del profesor fuera del aula: tareas y horas

\begin{tabular}{|l|c|}
\hline \multicolumn{1}{|c|}{ Tareas } & Horas \\
\hline 1. Organización de equipos & $3 \mathrm{~h}$ \\
\hline $\begin{array}{l}\text { 2. Diseño de la actividad, inserción en el programa, } \\
\text { material para el alumnado }\end{array}$ & $6 \mathrm{~h}$ \\
\hline 3. Batería de preguntas (diseño y presentación) & $16 \mathrm{~h}$ \\
\hline 4. Preparación de temas & $6 \mathrm{~h}$ \\
\hline 5. Solución de problemas & $3 \mathrm{~h}$ \\
\hline TOTAL & $34 \mathrm{~h}$ \\
\hline
\end{tabular}

La organización de equipos comprende la constitución de grupos heterogéneos de trabajo empleando las notas recogidas mediante la observación en el aula y las pruebas de referencia. El segundo apartado abarca la planificación de la actividad (normas, premios, distribución en sesiones, etc.), la inserción de la misma en el programa del curso atendiendo al tiempo disponible en el aula y la distribución del resto de actividades de evaluación continua, y también el tiempo dedicado a la preparación del material para el alumnado. El diseño de la batería de preguntas se llevó a cabo mediante un proceso de selección y adaptación de diversas fuentes, e inclusión de preguntas elaboradas por el profesor para ajustarse al material de trabajo y al nivel. En este apartado se incluye además el tiempo empleado en diseñar y elaborar las presentaciones con las preguntas del concurso. El apartado de preparación de temas comprende la preparación de las sesiones introductorias de cada tema por parte del profesor. Finalmente, el apartado de solución de problemas abarca las medidas tomadas para solventar dificultades previsibles o surgidas en el transcurso de las actividades (ver sección siguiente), e incluye el tiempo dedicado a realizar correcciones para cursos posteriores, sustituyendo o adaptando preguntas que resultaron ser ambiguas o que planteaban algún tipo de dificultad imprevista.

\subsubsection{Dificultades superadas}

La creación de la figura del ayudante y la introducción de un sistema de penalizaciones contribuyeron a aminorar los inconvenientes derivados del absentismo y del desigual número de alumnos en grupos y equipos. Por lo que respecta al sistema de penalizaciones, las faltas de asistencia descontarían a cada individuo 0,05 puntos de la puntuación final obtenida por su equipo. El docente consideró la penalización individual como una medida justa para el resto del equipo desde una óptica cooperativa (quien menos contribuye al conjunto, menos premio recibe), siendo a un tiempo razonablemente baja para no perjudicar en exceso a quien se ausentase ocasionalmente con causa justificada.

\subsubsection{Dificultades no superadas}

A fin de observar el comportamiento de los equipos sin influencia externa y anotar sus hábitos y estrategias de trabajo, no se proporcionó entrenamiento específico para afrontar la fase de preparación: el profesor únicamente explicó a los grupos que debían trabajar en equipo 
y prepararse entre todos, haciendo ver que el tiempo del que disponían era limitado, por lo que debían emplear el material proporcionado que estimasen conveniente en función de sus necesidades, y que su objetivo era asegurarse de que todos los miembros se familiarizaban con el tema y eran capaces de responder a preguntas de diferentes grados de dificultad sobre el mismo. El docente actuaría como supervisor de todo el proceso, resolviendo dudas que los equipos no fueran capaces de solucionar por sí mismos. Mientras los grupos trabajaban, el profesor observó y tomó nota del comportamiento, estrategias y hábitos de trabajo de los equipos, empleando como herramienta para sistematizar la recogida de datos un listado de parámetros y valores relativos a agrupamientos internos, asignación de roles, y turnos y tiempo de habla. De ello se desprende la constatación de una serie de problemas detectados tanto en la asignatura del grado como en la anterior de la diplomatura.

En general, y como era previsible, se observa que el alumnado carece de hábitos de trabajo cooperativo; los equipos no saben cómo desarrollar la responsabilidad individual dentro del grupo ni cómo distribuir la tarea haciendo que todos desempeñen un papel, al margen de contestar preguntas. Se verifica además una fuerte tendencia al individualismo y a la segregación: en 10 de los 14 equipos (el 71,4\%) los miembros trabajan en parejas, individualmente, o chicos y chicas por separado. Dichos equipos comenzaban la preparación leyendo la información teórica individualmente, en parejas o en grupos de 3, para a continuación realizar los ejercicios propuestos sin proceso de selección alguno, manteniendo los agrupamientos internos y, en caso de existir alguna duda, acudiendo al alumno de mayor nivel, el cual trabajaba en pareja o en grupo de 3 en 7 de los equipos, e individualmente en los 3 restantes. Es en su trabajo o en el de su subgrupo en donde se observa una cierta selección de actividades: 2 individuos y 4 subgrupos omiten algunas actividades referidas a teoría que no planteaba dudas. Cuando los equipos no se disgregaban en subgrupos o individuos trabajando por su cuenta, el procedimiento a seguir lo determinaba normalmente un alumno de mayor competencia y menor introversión, que asumía el rol de profesor leyendo el material para todos, solventando dudas y pidiendo respuestas a los ejercicios, mientras el resto del equipo le seguía. Este modo de interacción se observa en 4 de los 14 equipos (el 28,5\%), y en dos de ellos el rol de profesor lo compartían un alumno de mayor nivel y otro de buen nivel pero mayor extroversión. En 3 de los 4 equipos con rol de profesor se constata también una selección de actividades. Por último, es preciso señalar que uno de los equipos que trabajaba en subgrupos cambió sus hábitos de interacción en el último tema, asumiendo el rol de profesor un alumno que antes trabajaba individualmente. Este cambio podría deberse a la concienciación de que el trabajo aislado reportaría menos beneficios al individuo, dado que al final todo el equipo recibiría la misma puntuación.

Ningún equipo tuvo la iniciativa de poner en marcha una división de roles que fomentase la participación de todos y la responsabilidad individual, por ejemplo: un miembro lee la teoría para todos y comprueba que se entiende la gramática, vocabulario, etc.; otro hace preguntas a los demás o parafrasea para asegurarse de que todos entienden la teoría, otro selecciona los ejercicios en función del tiempo y las dudas planteadas, y distribuye su realización entre los compañeros, otro comprueba las soluciones y explica posibles dudas que surjan al realizar las actividades, y los roles se cambian en cada ronda. Se hace evidente que el alumnado carece de experiencia en cualquier distribución de roles distinta a la de profesor-alumnos (un profesor activo que selecciona, explica, pregunta, corrige y toma decisiones, y unos alumnos pasivos que obedecen, escuchan y responden), y que las alternativas han de ser introducidas, explicadas y practicadas en el aula. De otro modo, el trabajo 
de grupo acaba convirtiéndose en la suma descoordinada del trabajo individual, o cayendo en el reparto tradicional de papeles, en donde un alumno ejerce de profesor frente al resto.

Finalmente, el estudio de los hábitos de trabajo e interacción también constata fallos en la planificación de las tareas: en ninguno de los 14 equipos se pone en marcha una estrategia conjunta inicial que establezca prioridades para asegurar el mejor resultado con el tiempo disponible. El recurso empleado con más frecuencia para ganar tiempo es la omisión de actividades, y sólo ocasionalmente se reorganiza el material de trabajo (teoría y ejercicios) en función de las necesidades de los miembros del equipo: 4 equipos en la segunda ronda y 3 en la tercera alteraron significativamente la secuenciación del material presentado en la sesión introductoria.

\subsection{Evaluaciones y comentarios}

El alumnado participante en la experiencia respondió a una breve encuesta anónima de valoración que constaba de cuatro ítems: en primer lugar, se les solicitaba que calificaran de 1 a 10 la actividad realizada. A continuación, se les pedía que señalaran tres aspectos positivos y tres negativos de la actividad y que aportaran sugerencias de mejora. Finalmente, se preguntaba al alumnado si volvería a realizar una actividad similar en el aula de inglés. A continuación se recogen los resultados generales de las encuestas, con los porcentajes de respuestas más comunes y comentarios al respecto. Por motivos de simplicidad, se presentan los resultados unificados de los tres grupos interactivos.

En la encuesta participó el $87,3 \%$ del alumnado, otorgando a la actividad una calificación media de 8,29 puntos sobre 10. En la Tabla 3 se detallan los aspectos positivos y negativos señalados con más frecuencia:

Tabla 3. Valoración del alumnado: aspectos positivos y negativos de la actividad

\begin{tabular}{|l|c|l|c|}
\hline \multicolumn{1}{|c|}{ Aspectos positivos } & \multicolumn{1}{c|}{ Aspectos negativos } & \multicolumn{1}{c|}{} \\
\hline $\begin{array}{l}\text { Obliga a trabajar y a repasar lo } \\
\text { aprendido }\end{array}$ & $54,5 \%$ & $\begin{array}{l}\text { Diferencias de nivel entre los } \\
\text { equipos }\end{array}$ & $18,1 \%$ \\
\hline Fomenta la cooperación & $40 \%$ & $\begin{array}{l}\text { Presión individual /No se permite } \\
\text { contestar en equipo }\end{array}$ & $12,7 \%$ \\
\hline Diversión & $34,5 \%$ & Dependencia del equipo & $10,9 \%$ \\
\hline Facilita el aprendizaje & $25,4 \%$ & $\begin{array}{l}\text { El factor azar en el nivel de las } \\
\text { preguntas }\end{array}$ & $9 \%$ \\
\hline $\begin{array}{l}\text { Aumenta la motivación para } \\
\text { aprender }\end{array}$ & $12,7 \%$ & $\begin{array}{l}\text { Rivalidad /excesiva } \\
\text { competitividad de algún alumno/ } \\
\text { equipo }\end{array}$ & $9 \%$ \\
\hline $\begin{array}{l}\text { Formato original /actractivo para } \\
\text { aprender: dinámico /competitivo }\end{array}$ & $10,9 \%$ & Dificultad de las preguntas & $5,4 \%$ \\
\hline & & Poco tiempo de preparación & $3,6 \%$ \\
\hline
\end{tabular}


Resulta destacable que el comentario positivo más frecuente no se relaciona con el aspecto cooperativo, sino que tiene que ver con el trabajo y el repaso. La observación de que la actividad obliga a trabajar continuamente y a repasar lo aprendido demuestra que esta actividad contribuye a facilitar el aprendizaje continuado y la planificación del alumnado para realizar su trabajo, puesto que le obliga a una revisión periódica de contenidos. No obstante, dicho dato puede tener una lectura menos positiva, ya que también evidencia que el alumnado, tras un largo periodo de vida académica, no ha adquirido aún hábitos de trabajo continuo ni desarrollado convenientemente sus capacidades para planificar el trabajo autónomo, y por tanto continúa necesitando de pautas y guías externas (esto es, que se le siga indicando lo que tiene que hacer, cuándo y cómo). La actividad contribuye asimismo a desarrollar las capacidades para el trabajo en equipo, que el alumnado reconoce como beneficioso. Por otra parte, se señalan como elementos positivos el aspecto lúdico de la actividad y su formato cooperativo y competitivo, que parecen fomentar también la motivación para el aprendizaje.

Los aspectos negativos señalados más comúnmente derivan de las limitaciones ya señaladas a la hora de poner en marcha la actividad y del propio formato de la misma, que persigue precisamente la creación de lazos de dependencia entre los miembros del equipo y el fomento de la responsabilidad de todos. Al carecer del tiempo suficiente para formar grupos de trabajo realmente heterogéneos, se acusan las diferencias de nivel entre los equipos, como señala el $18 \%$ de los encuestados. Es evidente que más tiempo para conocer al alumnado y organizar grupos de trabajo es esencial para la formación de equipos heterogéneos fiables, y éstos son una parte fundamental de la actividad. Del mismo modo, dedicar tiempo a entrenar a los equipos para trabajar juntos asumiendo roles en turnos y aumentar el tiempo de preparación favorece la creación de equipos cohesionados y puede aminorar el sentimiento de dependencia "negativa" del equipo.

Por lo que respecta a las sugerencias de mejora, las más frecuentes fueron permitir una respuesta conjunta del equipo a las preguntas $(9 \%)$, aumentar las sesiones de preparación (7.2\%), ampliar las rondas a otros temas, por ejemplo, para el aprendizaje del vocabulario (7.2\%), y permitir que los alumnos eligieran los equipos (5,4\%). Por último, el $98,4 \%$ del alumnado afirmó que repetiría la actividad.

\subsection{Limitaciones, conclusiones del estudio y propuestas}

Como ya se ha indicado, las limitaciones principales de la actividad derivan del tiempo disponible en el aula y del elevado número de alumnos. No obstante, la experiencia llevada a cabo fue altamente satisfactoria para alumnado y profesorado, aunque la valoración positiva y el alto porcentaje de alumnado que la repetiría pueden haberse visto influidos por el hecho de que se procuró que todo el mundo saliera beneficiado de la actividad. Teniendo en cuenta la encuesta al alumnado y la percepción general del profesor de las interacciones posteriores en el aula, la actividad consiguió aumentar la interacción del alumnado y mejorar las relaciones interpersonales, aunque con resultados discretos. A raíz de la actividad, algunos alumnos inicialmente reticentes a participar en el aula, bien por su nivel de inglés o bien por su introversión, aumentaron su grado de participación en actividades de grupo, lo cual puede tomarse como indicativo de un aumento de su autoestima. Por otra parte, y aunque 
también de modo discreto, pudo constatarse un progreso en el rendimiento al comparar los resultados obtenidos en las calificaciones de referencia con la actuación en el concurso y con los resultados de la prueba final de la asignatura. En esta prueba se incluyeron cuestiones sobre los temas tratados en el concurso, que se encontraron entre las de mayor porcentaje general de aciertos, si se compara con pruebas similares realizadas en cursos en los que no se llevó a cabo la actividad.

Las propuestas de investigación futura pasan por trabajar con grupos reducidos de alumnos y tomar decisiones de planificación y evaluación que permitan incorporar estas actividades a la dinámica cotidiana del aula. Con respecto a la temática, en este caso se ha partido de contenidos gramaticales, pero como señala Casal (2002), las actividades cooperativas pueden utilizarse para desarrollar todas las destrezas presentes en el aula de lengua extranjera, al tiempo que se trabajan contenidos actitudinales y se mejora la atmósfera del aula.

\section{Conclusiones generales}

Experiencias de aprendizaje cooperativo como la descrita en el presente estudio evidencian aspectos mejorables en el proceso de adaptación a Bolonia. Por lo general, el alumnado que llega a la universidad no ha desarrollado adecuadamente sus capacidades de planificación de tareas y establecimiento de prioridades como parte de sus hábitos de trabajo autónomo, ni tampoco está preparado para interactuar en equipos de trabajo eficaces, lo cual no resulta extraño ya que a lo largo de su vida académica han primado el aprendizaje pasivo, memorístico e individual, así como el trabajo de grupo eminentemente competitivo y muchas veces desequilibrado. No obstante, las capacidades para el trabajo autónomo y para el trabajo en grupo parecen darse en gran medida por sentadas, cuando la realidad demuestra que no es así. Como consecuencia, y siendo estas capacidades de vital importancia en el EEES, dichas habilidades han de desarrollarse en la universidad, muchas veces a partir de cero, y debiendo compartir espacio con apretados programas de contenidos propios.

Sin duda, cualquier actividad de aprendizaje cooperativo constituye una experiencia que, bien realizada, reporta numerosos beneficios para el alumnado: éste aprende a trabajar realmente en equipo, a asumir responsabilidades, y adquiere habilidades sociales. Además, en este caso en particular, tiene la posibilidad de practicar la lengua extranjera y mejorar su nivel de competencia individual. A este respecto, es importante destacar que este tipo de actividades de acción colectiva posibilita además el enriquecimiento del enfoque comunicativo para acomodar los nuevos objetivos de aprendizaje apuntados por el MCER, surgidos de los cambios económicos y políticos experimentados en Europa desde la década del 2000. Como observa Puren (2004: 35-36), dichos objetivos se han trasladado desde el "intercambio más rápido y eficaz de informaciones", propio del enfoque comunicativo, hacia "la capacidad, para cada ciudadano europeo, de vivir y trabajar con extranjeros", esto es, la capacidad para la convivencia y, por tanto, para la "co-acción".

No obstante, como también se ha comprobado en el estudio, para surtir los efectos deseados este tipo de aprendizaje necesita un tiempo de asimilación y de maduración, así como dedicación dentro y fuera del aula, tanto por parte del profesor para formarse, pre- 
parar, planificar y ejecutar las actividades, como por parte del alumnado para entrenar y desarrollar sus habilidades para el trabajo en grupos cohesionados y eficaces. Como señala Escribano (1995: 96), para "trabajar con eficacia de manera cooperativa", es preciso, entre otros aspectos, proporcionar al alumnado "tiempo y espacio para compartir las características de esta estrategia”. El tiempo es precisamente un factor que los actuales planes de estudio no parecen favorecer, al haberse reducido tanto la duración de las titulaciones como la presencialidad en las aulas, destinándose gran parte de las horas de dedicación del alumnado al desarrollo de un "trabajo autónomo" para el que, sin embargo, parece carecer de herramientas en la práctica.

Por otra parte, el entrenamiento precisa asimismo de recursos humanos y materiales: como señalan León del Barco y Latas (2007), es necesario "configurar espacios, recursos, medios y horarios para adaptar a la universidad la metodología del aprendizaje cooperativo", y dicha adaptación no podrá realizarse "si los docentes universitarios acometen esta importante tarea en solitario o sin suficiente soporte institucional" (2007: 275). A este respecto, parece imprescindible contar con la colaboración, no sólo de otros docentes universitarios, sino también del profesorado de otras etapas educativas.

Por último, aunque quizá sea lo más importante, es esencial un cambio de actitud en el profesorado universitario con respecto a la docencia: recogiendo la opinión de León del Barco y Latas (2007: 275), dicho cambio se materializa en "una mayor preocupación por los procesos de enseñanza-aprendizaje y por el alumno". Uno de los principales escollos en este camino es, no obstante, y como bien denuncian Alfaro y Pérez (2011), el aún escaso reconocimiento a la labor y a la innovación docente en la carrera profesional del profesorado universitario en contraste con las tareas investigadoras, lo cual incide negativamente "a la hora de realizar un cambio en las costumbres docentes de los profesores" (2011: 42). La pregunta es si, a pesar de esta evidencia, el profesorado está dispuesto a dedicar más tiempo dentro y fuera del aula a que sus estudiantes aprendan a ser autónomos, responsables y grupalmente eficaces, contribuyendo de este modo a su formación humana integral o, en palabras de Escribano (1995: 92), a formar personas no sólo "más reflexivas e investigadoras", sino también más "humanistas" y "cooperativas".

\section{Bibliografía}

Alfaro Rocher, I. J. y Pérez Boullosa, A. (2011). "Un estudio acerca de la dedicación docente del profesorado universitario en el marco del Espacio Europeo de Educación Superior", en Revista Electrónica Interuniversitaria de Formación del Profesorado, 14, 4: 41-56.

Andreu Barrachina, Ll. y Sanz Torrent, M. (2010). "El juego-concurso de De Vries: una propuesta para la formación en competencias de trabajo en equipo en la evaluación", en Revista de Docencia Universitaria REDU, 8, 1: 121-41, disponible en http://redaberta.usc.es/redu, recuperado el 2 de octubre de 2013.

Area, I. y Martín, A. (2009). “Aprendizaxe cooperativa na universidade”, en Revista Galega do Ensino, 55: 73-77.

Barba, J.J., Martínez, S. y Torrego, L. (2012). "El proyecto de aprendizaje tutorado cooperativo. 
Una experiencia en el grado de maestra de educación infantil", en Revista de Docencia Universitaria REDU, 10, 1: 123-44, disponible en http://redaberta.usc.es/redu, recuperado el 2 de octubre de 2013.

Bernabé Villodre, M. (2012). "Interculturalidad y trabajo cooperativo en el aula de música de primaria", en J.I. Alonso, A. Escarbajal y R. Nortes (eds.), Experiencias de innovación en educación infantil y educación primaria. Murcia: Universidad, 61-68.

Cano Tornero, M.C. (2012). "Aprendizaje cooperativo en educación infantil: una experiencia innovadora desde la práctica educativa”, en J.I. Alonso, A. Escarbajal y R. Nortes (eds.), Experiencias de innovación en educación infantil y educación primaria. Murcia: Universidad, 11-18.

Casal, S. (2002). "El aprendizaje cooperativo en el aula de lengua extranjera", en G. López (ed.), Lenguas extranjeras: hacia un nuevo marco de referencia en su aprendizaje. Madrid: Secretaría General Técnica, Ministerio de Educación, Cultura y Deporte, 103-15.

DeVries, D. y Edwards, K. (1973). "Learning games and student teams: their effects on classroom process", en American Educational Research Journal, 4: 307-18.

DeVries, D. y Edwards, K. (1974). "Student teams and learning games: their effects on cross-race and cross-sex interaction", en Journal of Educational Psychology, 66: 741-49.

Escribano González, A. (1995). "Aprendizaje cooperativo y autónomo en la enseñanza universitaria”, en Enseñanza, 13: 89-102.

Fraile, A. (2008). "El aprendizaje cooperativo como metodología para el desarrollo de los ECTS: una experiencia de formación del profesorado de educación física", en Fuentes, 8: 22-35.

García, R., Traver, J. y Candela, I. (2001). Aprendizaje cooperativo: fundamentos, características y técnicas. Madrid: CCS.

Geronès, M. y Surroca, M. (1997). "Una experiencia de aprendizaje cooperativo en educación secundaria", en Aula de Innovación Educativa, 59: 49-53.

Goicoetxea, E. y Pascual, G. (2002). “Aprendizaje cooperativo: Bases teóricas y hallazgos empíricos que explican su eficacia”, en Educación XX1, 5: 227-47.

González, J. y Wagenaar, R. (eds.)(2006). Tuning Educational Structures in Europe II. Bilbao: U. de Deusto /U. de Groningen.

Instituto Cervantes (trad.)(2002). Marco común europeo de referencia para las lenguas: aprendizaje, enseñanza, evaluación, disponible en http://cvc.cervantes.es/ensenanza/biblioteca_ele/ marco/cve_mer.pdf, recuperado el 2 de octubre de 2013

Johnson, D. y Johnson, R. (1994). Learning Together and Alone: Cooperative, Competitive and Individualistic Learning. $4^{\mathrm{a}}$ ed. Needham Heights, MA: Allyn \& Bacon.

Johnson, D., Johnson, R. y Holubec, E. (1994). Cooperative Learning in the Classroom. Alexandria, VA: Association for Supervision and Curriculum Development.

León del Barco, B. y Latas Pérez, C. (2007). "La formación en técnicas de aprendizaje cooperativo del profesor universitario en el contexto de la convergencia europea", en Revista de Psicodidáctica, 12, 2: 269-78.

Lorenzo, M., Santos, M., Olveira, E. y Rodríguez, A. (2002). “Cooperar para aprender en un mundo de ordenadores", en Aula Abierta, 79: 15-30.

López Rúa, P. (2010). “Aprendizaxe cooperativa na universidade: a experiencia dun modelo híbrido na aula de idiomas", en Innovación Educativa, 20: 49-59.

Ovejero, A. (1990). El aprendizaje cooperativo: una alternativa eficaz a la enseñanza tradicional. Barcelona: Promociones y Publicaciones Universitarias. 
Puren, Ch. (2004). "Del enfoque por tareas a la perspectiva co-accional", en Porta Linguarum, 1: 31-36.

Serrano, J., González-Herrero, M., Pons, R., Serrano, I., Álvarez-Castellanos, J. y Hellmuth, O. (2008). "El aprendizaje cooperativo en el proceso de enseñanza y aprendizaje de las ciencias sociales: diseño de una actividad de aula y análisis de la interacción", en Iber: Didáctica de las Ciencias Sociales, Geografía e Historia, 58: 108-20.

Slavin, R. (1986). Using Student Team Learning. Baltimore, MD: John Hopkins University, Center for Research on Elementary and Middle Schools. 